\title{
Hitoshi lba: Evolutionary approach to machine learning and deep neural networks: neuro-evolution and gene regulatory networks
}

\author{
Springer, 2018, Hardcover, 245 pp, ISBN: 978-981-13-0199-5
}

\section{Petra Vidnerová ${ }^{1}$}

Published online: 7 March 2019

(c) Springer Science+Business Media, LLC, part of Springer Nature 2019

The book's title promises an evolutionary approach to machine learning and deep neural networks. In fact, it covers both an evolutionary approach to machine learning and machine learning approach to evolutionary computations. It presents the current trends in the research area connecting evolutionary algorithms (EAs) and machine learning (ML), and covers a wide range of various hybrid approaches benefiting from a synergy between EAs and ML.

The book is organised in six chapters, each chapter beginning with a short abstract and ending with a list of references. The first two chapters are introductory, covering the methods and algorithms (evolutionary computation, machine learning, deep learning) that are used throughout the next three chapters (3-5), which form the core of the book. The last chapter is a short conclusion. The great advantage of the book is the large number of figures, illustrations, and diagrams, making the content easy to understand.

The first chapter explains the fundamental principles of evolution and introduces evolutionary computation. The chapter is written in a way that it is understandable for beginners, and more experienced readers may skip it. The list of references at the end of Chapter 1 is far from complete. For example, when introducing genetic programming (GP) I would expect a reference to John Koza. (The corresponding works are cited in the Preface on page $\mathrm{v}$ instead.)

The second chapter covers selected topics from machine learning and can be skipped by readers with a machine learning background. Chapter 2 is a little bit heterogeneous, individual sections have different presentation styles. Some methods are described in more detail than others. For example, Gröbner bases (a mathematical theory that is needed later in Chapter 4, particularly for the algebraic approach to GP) got much more space than other methods, including deep neural networks (DNN). The reader should not expect to get a complete knowledge of the DNN

Petra Vidnerová

petra@cs.cas.cz

1 The Czech Academy of Sciences, Institute of Computer Science, Prague, Czech Republic 
domain, only selected topics are briefly introduced. For example, the explanation on fooling images and their generation (pp. 69-70) is very brief. This is in fact the content of one of Nguyen's papers [1] squeezed into hardly one page.

The third chapter promises the evolutionary approach to deep learning. The reader interested in deep learning may be disappointed by its short length. Also the neuroevolution that forms a part of Chapter 3 is not directly connected to up-todate deep neural networks, since it describes NEAT and hyperNEAT algorithms (Section 3.1.1) that date back to 2002. On the other hand, based on NEAT is the DeepNEAT algorithm that is used for optimisation of architecture of deep neural networks. This algorithm is mentioned on p. 89, unfortunately without any details.

In spite of its short length, Chapter 3 covers several interesting approaches based on evolution and deep neural networks, such as genetically optimised convolutional neural networks and feature construction using genetic programming. These are topics that appeared only very recently.

While in the third chapter there are evolutionary approaches used to enhance deep learning methods, the fourth chapter is focused on approaches where machine learning methods are used to improve the performance of evolutionary computation. Chapter 4 contains not only a description of the ML and EAs algorithms but also a lot of figures and tables documenting their performance. This gives the reader complete information about the individual algorithms. The algorithms and results are quite recent, which is confirmed by up-to-date references.

Last, but not least, is the chapter dealing with gene regulatory networks. This is a very interesting topic overlapping with bioinformatics. It is quite challenging to cover such a broad research field in only one chapter. Moreover real-world applications are included, among them experiments with a humanoid robot. A whole book could be written on this topic. Still, it gives the reader an idea of what is going on in this research area. The reader does not need any previous knowledge of gene regulatory networks, however I miss a more formal definition of a gene regulatory network at the beginning of Chapter 5 .

Iba's book serves as a good overview of current research trends connecting EAs and ML, as well as an inspiration for further research. It contains both theory and applications. It is hardly possible to squeeze all this to one thin ( $245 \mathrm{p})$ book. Therefore the individual sections are quite brief. Nevertheless, they should give a reader a general idea about the methods and their applicability, then, the interested reader may follow the references. The deep neural networks mentioned in the title definitely attracts attention. However, I would not recommend the book to someone who is focused mainly on deep learning. The topic is touched in the book, several evolutionary methods to deep learning are included, but still it covers only a fraction of what is done in this area.

Evolutionary Approach to Machine Learning and Deep Neural Networks is intended both for beginners and experienced researchers. The basis of evolutionary algorithms and common machine learning methods are introduced, so it is self explanatory. However for someone with no machine learning background I recommend having a machine learning course textbook at hand while reading it. Experts both from EAs and ML field will find the book beneficial. 


\section{References}

1. A. Nguyen, J. Yosinski, J. Clune, Deep neural networks are easily fooled: high confidence predictions for unrecognizable images, in IEEE Conference on Computer Vision and Pattern Recognition, CVPR 2015, Boston, MA, USA, June 7-12, pp. 427-436 (2015). https://doi.org/10.1109/ CVPR.2015.7298640

Publisher's Note Springer Nature remains neutral with regard to jurisdictional claims in published maps and institutional affiliations. 